\title{
Cardiac catheterization in pulmonary hypertension: doing it right, with a catheter on the left
}

\author{
Georg Hansmann ${ }^{1}$, Stuart Rich ${ }^{2}$, Bradley A. Maron ${ }^{3}$ \\ ${ }^{1}$ Department of Paediatric Cardiology and Critical Care, Hannover Medical School, Hannover, Germany; ${ }^{2}$ Northwestern University Feinberg \\ School of Medicine, Chicago, IL, USA; ${ }^{3}$ Division of Cardiovascular Medicine, Brigham and Women's Hospital, Department of Cardiology, Boston \\ VA Healthcare System, Boston, MA, USA \\ Correspondence to: Georg Hansmann, MD, PhD, FESC, FAHA. Department of Pediatric Cardiology and Critical Care, Hannover Medical School, \\ Carl-Neuberg-Str. 1, 30625 Hannover, Germany. Email: georg.hansmann@gmail.com.
}

Submitted May 05, 2020. Accepted for publication May 30, 2020.

doi: $10.21037 / \mathrm{cdt}-20-483$

View this article at: http://dx.doi.org/10.21037/cdt-20-483

\section{Introduction}

Elevated mean pulmonary artery pressure (mPAP) is the principal hemodynamic criterion for pulmonary hypertension (PH) (1-4) - a condition that is associated with increased clinical risk across a wide spectrum of diseases (4-7). Since increased mPAP may occur from a diverse number of risk factors and pathways $(1,4,5,8)$, comprehensive clinical phenotyping with accurate cardiopulmonary hemodynamic assessment by cardiac catheterization is needed. By doing so, cardiac catheterization is important for classifying patients correctly to avoid misdiagnosis, delayed diagnosis, or the administration of inappropriate therapy (9). Integrating the hemodynamic and clinical profiles of patients at point-of-care is often challenging (10), however, and one common dilemma is establishing the contribution of left heart disease (LHD) to PH (11). Catheterizing the right heart and pulmonary arterial circulation is essential to allow for accurate diagnosis of PH, including PH-LHD (Group $2 \mathrm{PH}$ ), and for follow-up assessments.

\section{Cardiopulmonary hemodynamic assessment in pulmonary hypertension}

While echocardiography is a valuable screening modality for $\mathrm{PH}$, Doppler estimates of PAP are inaccurate in many patients, and cannot be used to quantify right atrial (RA), pulmonary venous, left atrial (LA) or left ventricular (LV) pressures reliably $(1,8)$. However, in addition to right ventricular end-diastolic pressure (RVEDP), left ventricular end-diastolic pressure (LVEDP) is necessary to classify and prognosticate patients appropriately, while measurements of $\mathrm{PV}$ and LA pressure are reserved for selected patients with congenital/structural cardiovascular diseases. In addition to directly measuring PAP, vital information from right heart catheterization includes pulmonary blood flow and endexpiratory pulmonary artery wedge pressure (PAWP) as a commonly used surrogate of LVEDP. From these data, the mean end-diastolic transpulmonary pressure gradient (TPG) and pulmonary vascular resistance (PVR) can be calculated (Box 1). Both diastolic TPG (syn. DPG, diastolic pressure gradient) and PVR (indexed to body surface area in children) are used to assign patients into one of three cardiopulmonary hemodynamic subtypes: pre-capillary $\mathrm{PH}$, isolated-postcapillary (Ipc-PH), or combined pre- and postcapillary PH (Cpc-PH) (Box 1) (9,14).

The likelihood that PH may be due to LHD in a particular patient hinges, in part, on the hemodynamic assessment and classification. For example, isolated precapillary PH is incompatible with PH-LHD as the cause, but rather a manifestation of pulmonary arterial hypertension $(\mathrm{PAH})$, chronic obstructive pulmonary disease (COPD), thromboembolic disease, or other causes. However, treatment options differ widely by clinical PH subtype; therefore, inaccurate cardiopulmonary hemodynamic assessment has important ramifications on patient outcome. This includes the missed opportunity to (I) improve quality of life, (II) decrease hospitalization, and (III) increase lifespan in PAH, for example, and the introduction of unnecessary risk by prescribing $\mathrm{PAH}$-specific pharmacotherapies to patients for whom an indication is lacking (15). 
Box 1 Definitions

$\mathrm{PH}$, according to the most recent, 6th WSPH (Nice, 2018)

mPAP $>20 \mathrm{mmHg}$ in children $>3$ months of age at sea level

Pre-capillary PH (e.g., PAH)

$\mathrm{mPAP}>20 \mathrm{mmHg}$

PAWP $\leq$ or LVEDP $\leq 15 \mathrm{mmHg}^{*}$

$P V R \geq 3$ WU (PVR index $\geq 3$ WU. m $^{2}$ BSA in children) ${ }^{\#}$

Diastolic TPG (DPG) $\geq 7 \mathrm{mmHg}$ (adjunct criterion)

Isolated post-capillary $\mathrm{PH}$ (Ipc-PH) in adults, e.g., predominantly diastolic LV dysfunction (HFpEF)*

mPAP $>20 \mathrm{mmHg}$

PAWP or LVEDP >15 mmHg

PVR $<3 \mathrm{WU}$ in adults (PVR index $\left.<3 \mathrm{WU} \cdot \mathrm{m}^{2}\right)$

Diastolic TPG (DPG) $<7$ mmHg (adjunct criterion)

Combination of pre-capillary and post-capillary $\mathrm{PH}(\mathrm{Cpc}-\mathrm{PH})$ in adults*

mPAP $>20 \mathrm{mmHg}$

PAWP or left ventricular end-diastolic pressure (LVEDP) $>15 \mathrm{mmHg}$

$P V R \geq 3$ WU (PVR index $\geq 3$ WU. $\mathrm{m}^{2}$ in children)

$\mathrm{PAH}$

mPAP $>20 \mathrm{mmHg}$

PAWP or LVEDP $\leq 15 \mathrm{mmHg}^{*}$

$P V R \geq 3 \mathrm{WU}$ (PVR index $\geq 3 \mathrm{WU} \cdot \mathrm{m}^{2}$ in children), plus criteria for group $1 \mathrm{PH}$

Pulmonary Hypertensive Vascular Disease (PHVD)

For biventricular circulations

mPAP $>20 \mathrm{mmHg}$ and PVR $\geq 3 \mathrm{WU}$ (PVR index $\geq 3 \mathrm{WU} \cdot \mathrm{m}^{2}$ in children)

For circulations with cavopulmonary anastomosis (e.g., Fontan physiology)

Mean TPG $>6 \mathrm{mmHg}$ (calculate mPAP minus mLAP or PAWP) or PVR index $>3 \mathrm{WU} \cdot \mathrm{m}^{2}$

The classification of $\mathrm{PH}$ according to the World Symposium on $\mathrm{PH}$ (WSPH, Nice, 2018) (2). Detailed hemodynamic definitions of PH (e.g., value of the diastolic transpulmonary pressure gradient) can be found in the 2015 ESC/ERS guidelines (1), Hansmann G. J Am Coll Cardiol, 2017 (8) and Apitz et al. Heart, 2016 (12). It should be noted that even mildly elevated mPAP values (20-24 mmHg, prognostic threshold $17 \mathrm{mmHg}$ ) are independent predictors of poor survival in adults with PH (Douschan et al. AJRCCM 2018) (13). In adults, PVR is not indexed to body surface area (BSA). Please note that the BSA has a large impact on the absolute numbers of systemic blood flow index (Qsi, cardiac index), pulmonary blood flow index (Qsi) and PVR index (PVRi), especially in either severely cachectic or very obese patients. ", PVR is calculated as mTPG divided by Qp, PVR index is calculatd as mTPG divided by Qp indexed to BSA. The mTPG (syn. DPD) is a pressure difference and calculated as mPAP minus mLAP or PAWP. *, in many instances, it is useful to measure the PAWP simultaneously with the LVEDP. LVEDP, left ventricular end-diastolic pressure; PH, pulmonary hypertension; BSA, body surface area; mPAP, mean pulmonary artery pressure; mLAP, mean left atrial pressure; PAH, pulmonary artery hypertension; PCWP, pulmonary capillary wedge pressure (syn. PAWP, pulmonary artery wedge pressure); PVR, pulmonary vascular resistance; TPG, transpulmonary pressure gradient; WSPH, World Symposium on Pulmonary Hypertension. 


\section{Pathophysiology of PH-LHD}

The etiology of PH-LHD involves many disorders encountered commonly in adult clinical practice (1), as well as conditions common to pediatric populations (8). This includes (I) heart failure with preserved LV ejection fraction (HFpEF) (16-18) that is epidemic in the elderly, (II) HF with reduced LVEF (HFrEF), and (III) anatomical leftsided obstruction, particularly in children and young adults with congenital heart disease (CHD) (e.g., valvar aortic stenosis, coarctation of the aorta, obstructive hypertrophic cardiomyopathy, and others) $(1,4,8,19)$. Among patients with PH-LHD, the Cpc-PH subgroup has emerged as a distinct clinical phenotype, which is defined by a diastolic TPG $\geq 7$ $\mathrm{mmHg}$ and/or PVR $>3 \mathrm{WU}\left(>3 \mathrm{WU} \cdot \mathrm{m}^{2}\right.$ body surface area in children) with PAWP or LVEDP $\geq 15 \mathrm{mmHg}$ (1).

LV dysfunction is not only a cause of PH-LHD, but is also an under-recognized complication of advanced $\mathrm{PH}$, including PAH (8,20-22). Severe (suprasystemic) right ventricular (RV) hypertension promotes a septal leftward shift and ventricular septal interdependence (20). Although early $\mathrm{PH}$ expert consensus guidelines allowed for direct $\mathrm{LV}$ hemodynamic assessment on a case-by-case basis, specific recommendations remain lacking. Current debate in the field seems to focus mainly on the need for cardiac catheterization in the assessment of $\mathrm{PH}$ at clinical follow-up $(23,24)$.

\section{Challenges to interpreting cardiopulmonary hemodynamics in PH-LHD}

Determining the hemodynamic PH classification can be challenging in practice. Elevated PAWP may not be evident in PH-LHD patients treated with diuretics or may be observed only during physical activity (25), as just two common but complex example scenarios. Furthermore, valvar or subvalvar aortic stenosis (LVOTO), (residual) coarctation of the aorta, and/or LA hypertension due to an intracardiac shunt are important considerations since management of PH-LHD. Treatment in these scenarios is vastly different than for other forms of $\mathrm{PH}$ LHD and emphasizes relieving the left-sided obstruction/ regurgitation by surgery or catheter-based intervention. Notwithstanding these special circumstances, two common practice patterns hamper the hemodynamic data assessment and interpretation for patients at-risk for PH-LHD: nonstandardized timing of cardiac catheterization and the method for measuring/estimating LVEDP.

\section{Timing of cardiac catheterization: opportunity for standardizing the approach to PH-LHD diagnosis}

Guidelines on the timing of right heart catheterization in patients with risk factors for PH-LHD are lacking, and practice patterns vary by geography, experience of provider, and patient population $(7,26)$. Of note, early diuretic administration is common in incident LHD patients, and may decrease PAWP level to a value that could imply "isolated pre-capillary PH". A possible alteration of PAWP bears on both patient classification and prognosis since major differences in outcome are reported between patients with similar mPAP and PVR stratified by normal $v$ s. elevated PAWP (13). Yet, in LHD common indications for cardiac catheterization often involve extreme scenarios: cardiogenic shock and end-stage heart failure or, conversely, confirming normal PAWP in stable patients with equivocal physical examination and biochemical data on volume status.

We propose that an initial right heart catheterization should ideally be performed in patients suspected of PHLHD while in the symptomatic state. If diuresis is required first, then confrontational fluid challenge or exercisecardiac catheterization $v$ s. invasive cardiopulmonary exercise test may be indicated $(8,9)$. If these maneuvers are not possible, then the effect of diuresis on interpreting hemodynamics must be considered, including the possibility that elevated PAWP was present prior to treatment. On follow-up assessment, cardiac catheterization in adult PHLHD patients should be reserved to determine if persistent dyspnea is explained by elevated PAWP despite stable body weight, medication adherence, and low sodium/cardiac diet. In children and adults with congenital/structural heart disease (e.g., cardiovascular shunts, small left-sided structures), comprehensive hemodynamic assessment requires right and left heart catheterization, and acute vasoreactivity testing in those with pre-capillary $\mathrm{PH}$ $(1,4,8,12)$. Diagnostic cardiac catheterization is usually postponed in very small infants or in sick patients until they are hemodynamically stabilized (e.g., via balloon dilation for severe valvular aortic stenosis; intensive care in myocarditis or extreme prematurity).

\section{Targeted role for left heart catheterization in PH-LHD diagnosis}

In our view, the diagnostic accuracy of PH-LHD will increase with direct measurement of LVEDP. We propose 


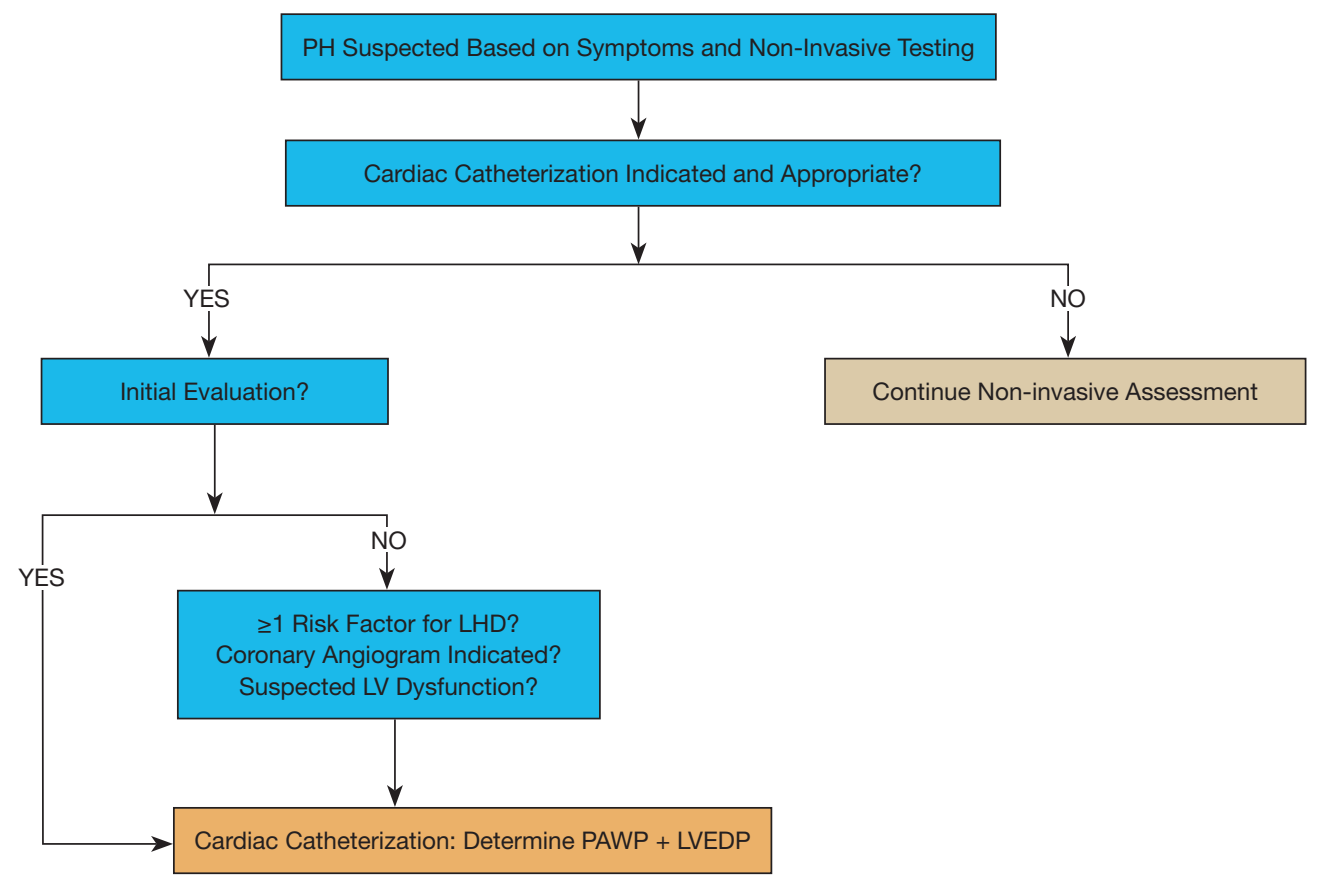

Figure 1 Proposed algorithm for incorporating left heart catheterization into the diagnostic approach to pulmonary hypertension. LHD, left heart disease; LV, left ventricle; PAWP, pulmonary artery wedge pressure; LVEDP, left ventricular end-diastolic pressure.

that left heart catheterization, defined by LV intracardiac pressure measurement and-if indicated-coronary angiography, should become an integrative part of the initial $\mathrm{PH}$ evaluation, as well as the follow-up assessment in selected patients (Figure 1). In addition, aortic pressure measurements (ascending aorta, descending thoracic aorta) can easily be conducted to determine the systolic gradient in LVOT obstruction, including supra- and subvalvular aortic stenosis, or coarctation of the aorta as a cause for LV pressure load and/or systemic arterial hypertension. Moreover, simultaneous aortic pressure measurements allow for more accurate determination of $\mathrm{mPAP} / \mathrm{mSAP}$ ratio, and invasive monitoring of systemic arterial perfusion.

Our rationale is based on several points. First, inaccurate PAWP results are common due to 'overwedging' of the catheter, large "V waves" (often in the setting of mitral valvular regurgitation or impaired LV compliance) or lack of PAWP readings in end-expiration, among other technical reasons and conditions (e.g., pulmonary veno-occlusive disease, chronic thromboembolic $\mathrm{PH})(9,27)$.

Second, simultaneous PAWP and LVEDP measurements minimize technical errors. Even when cardiac catheterization is performed conscientiously (including re-zeroing, re-wedging), we frequently encounter $\mathrm{PH}$ patients with a significant discrepancy between PAWP and LVEDP $(>5 \mathrm{mmHg}$ ) when measured simultaneously. This discrepancy (PAWP > LVEDP) is often evident in patients with parenchymal/interstitial lung disease, and may also occur in pulmonary vein disease or mitral valve stenosis. On the other hand, under conditions in which the a-wave is attenuated or absent, such as atrial fibrillation, the LVEDP may underestimate PAWP. Indeed, unrecognized LV diastolic dysfunction, mitral stenosis or pulmonary vein disease may explain why some $\mathrm{PH}$ patients do not tolerate pulmonary vasodilators that are associated with substantial increases in pulmonary blood flow.

Third, there is limited consensus on ideal PAWP recording in patients with respiratory undulation, and right heart catheterization-only does not discriminate pulmonary venous from LA hypertension, with or without increased LVEDP. While PAWP measurements reflect luminal pressure in the larger pulmonary veins, there is increasing evidence in support of pulmonary venule involvement in both PAH and PH-LHD.

Fourth, cardiopulmonary hemodynamics and physiology differ substantially between patients whose $\mathrm{PAH}$ is either idiopathic or due to CHD. In the latter group, for example, a RV decompressing shunt (patent ductus arteriosus, 
ventricular septal defect) alleviates septal shifting and increases cardiac output, and consequently is associated with favourable outcome $(8,28)$. In these scenarios, left heart catheterization, with simultaneous PAWP (or LAP) and LVEDP measurements, provides insightful information on the postcapillary, pulmonary venous - left heart hemodynamics.

Finally, left heart catheterization permits diagnostic coronary angiography or hemodynamic assessment of valvular disease, which, in turn, has important potential implications on clinical care for patients with $\mathrm{PH}$ and mitral/aortic valve disease, or coronary artery stenosis which can manifest as effort related dyspnea $(1,9)$.

\section{Evidence favouring LVEDP assessment in patients at-risk for PH-LHD}

The extent to which PAWP and LVEDP are discordant in referral populations has been published widely. In one single-center recent study focusing on 2,270 patients undergoing combined right and left heart catheterization, only a moderate correlation was observed between PAWP and LVEDP ( $\mathrm{r}=0.6)$ (29). Numerous clinical variables associated commonly with PH-LHD limited the accuracy of PAWP relative to LVEDP, such as increased age and LA diameter (29). A prospective study of 61 patients with suspected PH recorded PAWP and LVEDP subsequently during combined right and left heart catheterization (27). The authors found that the common practice of using PAWP-digital (mean monitor PAWP; PAWPdigital $8.0 \mathrm{mmHg}$ ) instead of PAWP in end expiration (PAWP-end Exp; $13.0 \mathrm{mmHg}$ ) results in a significant underestimation of LVEDP-end expiration (PAWPdigital $8.0 \mathrm{mmHg} v s$. LVEDP-end $\operatorname{Exp} 12.4 \mathrm{mmHg}$; $\mathrm{P}<0.05)(27)$. This discordance between PAWP-digital and LVEDP misclassified $~ 30 \%$ of these patients with $\mathrm{PH}$ LHD as to have PAH (27). When PAWP and LVEDP are recorded simultaneously, a significant discrepancy between PAWP and LVEDP should prompt adjustments (rezeroing, -positioning, -wedging, and contralateral PAWP measurements). If the discrepancy persists, searches for the cause are indicated (selective pulmonary vein angiography, pulmonary artery wedge angiography, simultaneous LA and LVEDP recordings, chest computed tomography, etc.). In patients with chronic thromboembolic $\mathrm{PH}$, there is no way of knowing if a thrombus is present distal to the wedged PA catheter.

Data from population studies in adults suggest that the complication rate associated with left heart catheterization (including coronary angiography) is low in adults $(\sim 0.7 \%)(30)$. However, the risks and benefits of left heart catheterization must be individualized to each patient and especially pediatric procedures should be performed in experienced $\mathrm{PH}$ centers. It should be noted that the addition of left to right heart catheterization does not fully characterize cardiopulmonary hemodynamics, since LA or pulmonary venule pressure are not measured directly (unless there is an interatrial communication). Nevertheless, in our view, left heart catheterization is a valuable but underutilized tool for the initial evaluation of many $\mathrm{PH}$ patients (Figure 1), particularly those with HFpEF (or other conditions that predispose to Ipc-PH), Cpc-PH, and/or structural heart disease/CHD; left heart catheterization should also be considered in follow-up assessments in selected patients, for example, those with previously documented elevated left-sided filling pressures (LVEDP). With respect to clinical follow-up of $\mathrm{PH}$ patients, we align with the view that is 'pro-cardiac catheterization' [right heart + right and left pulmonary arteries (23), or combined right/left heart catheterization] as fundamental to $\mathrm{PH}$ diagnosis, irrespective of $\mathrm{PH}$ etiology, although this issue has been under a current debate $(23,24)$.

Further clinical data are needed that integrate cardiac catheterization results with non-invasive diagnostic modalities, such as echocardiography, cardiac MRI with TWIST angiography, or new circulating biomarkers, to phenotype and risk stratify patients early in the natural course of $\mathrm{PH}$ (31). In this way, the ideal timing and frequency of cardiac catheterization in $\mathrm{PH}$ may become clearer.

\section{Conclusions}

In summary, PH-LHD (group $2 \mathrm{PH}$ ) is a complex cardiopulmonary hemodynamic condition that is not clarified solely by LV structure or function. Determining that elevated pulmonary artery pressure and increased left heart pressure is present requires catheterization of the right heart and bilateral pulmonary arteries. Cardiac catheterization should be performed initially in symptomatic $\mathrm{PH}$ patients stable enough to undergo the procedure. Additionally, left heart catheterization, including direct measurement of LVEDP simultaneously with PAWP, offers a diagnostic advantage to PAWP alone for identifying patients with either pulmonary venous hypertension, significant lung disease, or mitral stenosis. Coronary angiography may be indicated in select cases when $\mathrm{PH}$ is 
confirmed. Overall, future consensus statements are needed that consider strengthening the indication for left heart catheterization in the diagnostic evaluation of $\mathrm{PH}$ patients by clarifying specific scenarios for which this approach is warranted.

\section{Acknowledgments}

Funding: GH receives financial support from the German Research Foundation (DFG; HA4348/2-2 and HA4348/6-2 KFO311), the Federal Ministry of Education and Research (BMBF ViP+ program 03VP08053; BMBF 01KC2001B) and the European Pediatric Pulmonary Vascular Disease Network (www.pvdnetwork.org). BAM is supported by 1R01HL139613-01; R21HL145420; the Boston Biomedical Innovation Center (B-BIC), and the Cardiovascular Medical Research and Education Foundation (CMREF).

\section{Footnote}

Provenance and Peer Review: This article was commissioned by the Guest Editors (Martin Koestenberger, HarmJan Bogaard and Georg Hansmann) for the series "Right Ventricular Dysfunction" published in Cardiovascular Diagnosis and Therapy. The article was sent for external peer review organized by the Editor-in-Chief and the editorial office.

Conflicts of Interest: All authors have completed the ICMJE uniform disclosure form (available at http://dx.doi. org/10.21037/cdt-20-483). The series "Right Ventricular Dysfunction" was commissioned by the editorial office without any funding or sponsorship. GH served as the unpaid Guest Editors of the series. BAM reports personal fees from Actelion, outside the submitted work. The authors have no other conflicts of interest to declare.

Ethical Statement: The authors are accountable for all aspects of the work in ensuring that questions related to the accuracy or integrity of any part of the work are appropriately investigated and resolved.

Open Access Statement: This is an Open Access article distributed in accordance with the Creative Commons Attribution-NonCommercial-NoDerivs 4.0 International License (CC BY-NC-ND 4.0), which permits the noncommercial replication and distribution of the article with the strict proviso that no changes or edits are made and the original work is properly cited (including links to both the formal publication through the relevant DOI and the license). See: https://creativecommons.org/licenses/by-nc-nd/4.0/.

\section{References}

1. Galie N, Humbert M, Vachiery JL, et al. 2015 ESC/ERS Guidelines for the diagnosis and treatment of pulmonary hypertension: The Joint Task Force for the Diagnosis and Treatment of Pulmonary Hypertension of the European Society of Cardiology (ESC) and the European Respiratory Society (ERS): Endorsed by: Association for European Paediatric and Congenital Cardiology (AEPC), International Society for Heart and Lung Transplantation (ISHLT). Eur Heart J 2016;37:67-119.

2. Simonneau G, Montani D, Celermajer DS, et al. Haemodynamic definitions and updated clinical classification of pulmonary hypertension. Eur Respir J 2019;53:1801913.

3. Rosenzweig EB, Abman SH, Adatia I, et al. Paediatric pulmonary arterial hypertension: updates on definition, classification, diagnostics and management. Eur Respir J 2019;53:1801916.

4. Hansmann G, Koestenberger M, Alastalo TP, et al. 2019 Updated Consensus Statement on the Diagnosis and Treatment of Pediatric Pulmonary Hypertension. The European Pediatric Pulmonary Vascular Disease Network (EPPVDN), endorsed by AEPC, ESPR and ISHLT. J Heart Lung Transplant 2019;38:879-901.

5. Galie N, Channick RN, Frantz RP, et al. Risk stratification and medical therapy of pulmonary arterial hypertension. Eur Respir J 2019;53:879-901.

6. Hansmann G, Meinel K, Bukova M, et al. Selexipag for the treatment of children with pulmonary arterial hypertension: first multicenter experience in drug safety and efficacy. J Heart Lung Transplant 2020;39:695-706.

7. Hasan B, Hansmann G, Budts W, et al. Challenges and Special Aspects of Pulmonary Hypertension in Middle to Low Income Regions. J Am Coll Cardiol 2020;75:2463-77.

8. Hansmann G. Pulmonary hypertension in infants, children, and young adults. J Am Coll Cardiol 2017;69:2551-69.

9. Rosenkranz S, Gibbs JS, Wachter R, et al. Left ventricular heart failure and pulmonary hypertension. Eur Heart J 2016;37:942-54.

10. Frost A, Badesch D, Gibbs JSR, et al. Diagnosis of pulmonary hypertension. Eur Respir J 2019;53:1801904.

11. Vachiery JL, Tedford RJ, Rosenkranz S, et al. Pulmonary hypertension due to left heart disease. Eur Respir J 
2019;53:1801897.

12. Apitz C, Hansmann G, Schranz D. Hemodynamic assessment and acute pulmonary vasoreactivity testing in the evaluation of children with pulmonary vascular disease. Expert consensus statement on the diagnosis and treatment of paediatric pulmonary hypertension. The European Paediatric Pulmonary Vascular Disease Network, endorsed by ISHLT and DGPK. Heart 2016;102 Suppl 2:ii23-ii9.

13. Douschan P, Kovacs G, Avian A, et al. Mild Elevation of Pulmonary Arterial Pressure as a Predictor of Mortality. Am J Respir Crit Care Med 2018;197:509-16.

14. Palazzini M, Dardi F, Manes A, et al. Pulmonary hypertension due to left heart disease: analysis of survival according to the haemodynamic classification of the 2015 ESC/ERS guidelines and insights for future changes. Eur J Heart Fail 2018;20:248-55.

15. Maron BA, Ryan JJ. A Concerning Trend for Patients With Pulmonary Hypertension in the Era of EvidenceBased Medicine. Circulation 2019;139:1861-4.

16. Luscher TF. Heart failure with preserved ejection fraction: unravelling an enigma. Eur Heart J 2018;39:2763-6.

17. Lam CSP, Voors AA, de Boer RA, et al. Heart failure with preserved ejection fraction: from mechanisms to therapies. Eur Heart J 2018;39:2780-92.

18. Obokata M, Olson TP, Reddy YNV, et al. Haemodynamics, dyspnoea, and pulmonary reserve in heart failure with preserved ejection fraction. Eur Heart J 2018;39:2810-21.

19. Covella M, Rowin EJ, Hill NS, et al. Mechanism of Progressive Heart Failure and Significance of Pulmonary Hypertension in Obstructive Hypertrophic Cardiomyopathy. Circ Heart Fail 2017;10:e003689.

20. Friedberg MK. Imaging Right-Left Ventricular Interactions. JACC Cardiovasc Imaging 2018;11:755-71.

21. Burkett DA, Patel SS, Mertens L, et al. Relationship between left ventricular geometry and invasive hemodynamics in pediatric pulmonary hypertension. Circ Cardiovasc Imaging 2020;13:e009825.

22. Koestenberger M, Hansmann G. Left ventricular

Cite this article as: Hansmann G, Rich S, Maron BA. Cardiac catheterization in pulmonary hypertension: doing it right, with a catheter on the left. Cardiovasc Diagn Ther 2020;10(5):17181724. doi: $10.21037 / \mathrm{cdt}-20-483$ geometry and near-simultaneous invasive hemodynamics in pediatric pulmonary hypertension. Circ Cardiovasc Imaging 2020;13:e010787.

23. Humbert M, Weatherald J. Right heart catheterisation is still a fundamental part of the follow-up assessment of pulmonary arterial hypertension. Eur Respir J 2018;52:1800738.

24. Howard LS. Is right heart catheterisation still a fundamental part of the follow-up assessment of pulmonary arterial hypertension? The argument against. Eur Respir J 2018;52:1800996.

25. Naeije R, Saggar R, Badesch D, et al. Exercise-Induced Pulmonary Hypertension: Translating Pathophysiological Concepts Into Clinical Practice. Chest 2018;154:10-5.

26. Maron BA, Ryan JJ. Treatment differences in pulmonary arterial hypertension management. Pulm Circ 2016;6:619.

27. Ryan JJ, Rich JD, Thiruvoipati T, et al. Current practice for determining pulmonary capillary wedge pressure predisposes to serious errors in the classification of patients with pulmonary hypertension. Am Heart J 2012;163:589-94.

28. Hansmann G, Apitz C, Abdul-Khaliq H, et al. Executive summary. Expert consensus statement on the diagnosis and treatment of paediatric pulmonary hypertension. The European Paediatric Pulmonary Vascular Disease Network, endorsed by ISHLT and DGPK. Heart 2016;102 Suppl 2:ii86-ii100.

29. Hemnes AR, Opotowsky AR, Assad TR, et al. Features Associated with Discordance between Pulmonary Arterial Wedge Pressure and Left Ventricular End Diastolic Pressure in Clinical Practice: Implications for Pulmonary Hypertension Classification. Chest 2018;154:1099-107.

30. West R, Ellis G, Brooks N, et al. Complications of diagnostic cardiac catheterisation: results from a confidential inquiry into cardiac catheter complications. Heart 2006;92:810-4.

31. Maron BA, Brittain EL, Choudhary G, et al. Redefining pulmonary hypertension. Lancet Respir Med 2018;6:168-70. 